\title{
De Tayeb Salih à Abdelwahab Meddeb : Saison de la migration vers le Nord ou vers l'orientalisme?
}

From Tayeb Salih to Abdelwahab Meddeb: Saison de la migration vers le Nord or Towards Orientalism?

\section{Ridha Boulaâbi}

\section{OpenEdition}

\section{Journals}

Édition électronique

URL : http://journals.openedition.org/recherchestravaux/1764

DOI : $10.4000 /$ recherchestravaux.1764

ISSN : 1969-6434

\section{Éditeur}

UGA Éditions/Université Grenoble Alpes

\section{Édition imprimée}

ISBN : 978-2-37747-165-2

ISSN : 0151-1874

Référence électronique

Ridha Boulaâbi, «De Tayeb Salih à Abdelwahab Meddeb : Saison de la migration vers le Nord ou vers l'orientalisme? », Recherches \& Travaux [En ligne], 95 | 2019, mis en ligne le 05 décembre 2019, consulté le 08 avril 2021. URL : http://journals.openedition.org/recherchestravaux/1764 ; DOI : https:// doi.org/10.4000/recherchestravaux.1764

Ce document a été généré automatiquement le 8 avril 2021.

(c) Recherches \& Travaux 


\title{
De Tayeb Salih à Abdelwahab Meddeb : Saison de la migration vers le Nord ou vers l'orientalisme?
}

\author{
From Tayeb Salih to Abdelwahab Meddeb: Saison de la migration vers le \\ Nord or Towards Orientalism?
}

Ridha Boulaâbi

1 À partir d'une étude comparative de deux traductions en français de موسم الهجرة إلى الشمال (Saison de la migration vers le Nord), un grand classique de la littérature arabe des années soixante de l'écrivain soudanais Tayeb Salih ${ }^{1}$, cet article se propose de montrer comment certains choix, certaines décisions prises par les traducteurs révèlent des postures qui vont au-delà du simple fait de traduire. En effet, au cœur de l'espace du texte traduit, se joue une bataille en lien avec la discipline orientaliste.

2 Tayeb Salih publie d'abord son récit en plusieurs épisodes dans le magazine libanais

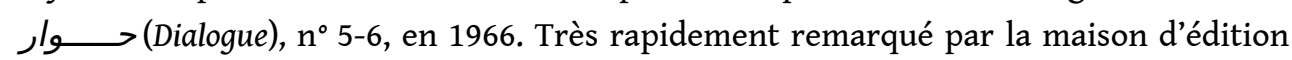
arabophone Dar Al-'Awdah, le roman sort la même année sous le titre موسم الهجرة إلى الشمال (Saison de la migration vers le Nord). Considéré comme l'un des chefs-d'œuvre de la littérature arabe contemporaine, l'histoire propose une relecture de l'orientalisme occidental et ses représentations de l'ailleurs musulman qui passe par la fiction. À partir d'un emboîtement de récits, Tayeb Salih retrace le parcours tumultueux de Mustapha Saïd, un jeune et brillant étudiant soudanais, exilé en Angleterre pour ses études universitaires. Pour explorer la complexité des rapports entre Orient et Occident en période coloniale, le narrateur se focalise en particulier sur les relations passionnelles voire meurtrières qu'entretient Mustapha Saïd avec les jeunes femmes anglaises. Le roman peut être lu, en ce sens, comme un essai dénonçant d'une manière très subtile l'orientalisme, l'impérialisme anglais et ses ravages, aussi bien en Afrique qu'en Angleterre. 
3 La première version traduite en français, de Fady Noun, offre au lecteur français une vision complètement édulcorée de l'imaginaire oriental sur l'occident, gommant presque systématiquement les critiques de l'orientalisme qui rythment le texte arabe. Mais la seconde version, d'Abdelwahab Meddeb, rétablit les passages coupés et permet de se rendre compte du rôle important jouée par la fiction arabe dans la déconstruction du savoir orientaliste, quelques années avant la publication de Orientalism d'Edward Saïd, en 1978. En replaçant les deux versions dans le contexte intellectuel français et européen avant et après le tournant saïdien, cette étude ambitionne, d'une part, de contribuer à la relecture de l'héritage orientaliste à partir du domaine arabe, lequel domaine a certainement nourri la critique de Saïd. D'autre part, elle propose des pistes de réflexion sur les répercussions qu'ont pu avoir ces débats sur l'œuvre littéraire d'un écrivain et essayiste comme Abdelwahab Meddeb.

\section{Contextes}

4 La fin des années soixante marque le début de tout un travail de décolonisation culturelle visant à relire l'héritage colonial européen du point de vue des peuples vaincus. Ces études qui se sont implantées dès 1967 à Leeds au Royaume-Uni, puis au Danemark, en Belgique dès 1970, ont contribué, par leur diffusion rapide, à la publication, en 1978, de L'Orientalisme d'Edward Saïd, aux États-Unis. C'est dans ce contexte de foisonnement intellectuel favorisant l'émergence des théories postcoloniales dans le monde anglo-saxon que paraît très rapidement, dès 1969, la première traduction en anglais de Saison de la migration vers le Nord, de Tayeb Salih, sous le titre Season of Migration to the North ${ }^{2}$, dans laquelle Denys Johnson-Davies, le traducteur du roman arabe, est très proche du texte d'origine.

Quant à la première traduction française du même roman publiée par Fadi Noun en 1972 chez Sindbad ${ }^{3}$, intitulée Le Migrateur, elle s'inscrit dans un contexte intellectuel tout autre. Comme l'explique bien Thomas Brisson dans ses travaux sur les épistémologies postcoloniales, les études orientales en France ont connu une évolution plus lente qu'ailleurs ${ }^{4}$. Malgré la critique ouverte de l'orientalisme scientifique formulée pour la première fois par Anouar Abdel-Malek dans son article "L'orientalisme en crise $^{5}$ ", paru en 1963 dans la revue Diogène, il faut attendre la création, en 1968, de départements d'arabe décentralisés pour voir émerger des recherches sur le monde arabe contemporain. En 1971, ces recherches se déploient au sein de formations en sciences sociales, sous l'impulsion du CNRS et aux dépens de l'orientalisme classique, resté cantonné dans l'étude de l'Orient ancien, des langues orientales et de la religion. En somme, si le plaidoyer d'Anouar Abdel-Malek vise les rapports étroits entre la domination coloniale et l'érudition orientaliste, il ne s'attaque pas frontalement, comme le fait remarquer Thomas Brisson, aux autres facettes de l'orientalisme occidental, celui des missionnaires, des militaires, des écrivains, des peintres ou encore des musiciens. Sa critique pointe surtout du doigt l'organisation du savoir au sein de l'Institut d'études islamiques de la Sorbonne, jugé réfractaire à toute ouverture sur l'Orient contemporain. C'est donc dans ce contexte marqué à la fois par une ouverture à la culture arabe contemporaine et en même temps par une quasi absence de mise en question frontale des structures de l'orientalisme classique, que la première version du roman de Tayeb Salih sort en France. Parfaitement représentative 
de ce contexte, cette version de 1972 ne comporte aucune critique de l'imaginaire orientaliste occidental, le texte ayant été complètement retravaillé par le traducteur.

La comparaison des deux versions traduites en français nous permettra de montrer en particulier les coupes opérées par le premier traducteur, qui ont pour conséquence de gommer la portée critique de l'œuvre à l'égard de l'orientalisme. Cette étude révèlera, entre autres, les conflits politiques et épistémologiques à l'œuvre entre les langues française et arabe.

\section{Traduction en cul-de-jatte}

7 Lorsqu'en 1983, les éditions Sindbad décident de publier une nouvelle traduction du roman soudanais et qu'elles en confient la tâche à Abdelwahab Meddeb, celui-ci, sans faire table rase complète de la version de Fadi Noun, décide d'être plus proche du texte source. Il justifie ce travail de remaniement dès l'ouverture du livre, de la manière suivante :

Sindbad a publié ce roman en 1972, sous le titre Le Migrateur. Cette première traduction, de Fady Noun, n'était pas intégrale. J'ai donc transmis l'ampleur et l'intégralité du texte original. Mon souci étant de rester fidèle à l'enchaînement et à la temporalité du texte arabe, c'est-à-dire à son rythme, sa musicalité. Le roman est rendu à son titre littéral: Saison de la migration vers le Nord. Le texte arabe utilisé pour ce travail a été publié à Tunis, en 1978, par Sud Éditions. (A. M., p. 7)

Comme nous l'avons évoqué précédemment, une grande partie des passages tronqués dialogue tantôt directement, tantôt d'une manière implicite, avec le discours orientaliste occidental que le romancier ne cesse de délégitimer en passant par la fiction. En ce sens, la tâche que se donne Abdelwahab Meddeb ne consiste pas uniquement à reconstituer l'intégralité du texte arabe en français. Plus important encore, il s'agit pour lui, en tant que traducteur, de contribuer aux débats sur les représentations occidentales de l'Orient qui animent la scène intellectuelle et universitaire occidentale depuis la publication du livre de Saïd. Cette contribution passe par l'intégration, dans ces débats, d'une voix littéraire postcoloniale dont le message a été écarté, pour ne pas dire complètement déformé. Une telle déformation se manifeste par l'absence, dans la première version française, d'un certain nombre de phrases, de paragraphes, voire même de pages entières, ainsi que de références très importantes quant à la compréhension de la thèse profonde défendue par le roman. Cette trahison commence par l'effacement d'expressions symboliquement fortes comme le révèle la comparaison entre les deux passages suivants (les expressions supprimées sont mises en valeur en gras) :

J'ouvris un cahier et lus sur la première page: "histoire de ma vie - Moustafa Saïd », Sur la page suivante, la dédicace : « À ceux dont le regard est franc, la parole est simple, pour qui les choses sont blanches ou noires ». Je feuilletai le reste du cahier... (F. N., p. 115)

J'ouvris un cahier et lus sur la première page: «histoire de ma vie - Moustafa Saïd », Sur la page suivante, la dédicace : « À ceux qui voient d'un regard net, à ceux qui parlent d'une voix catégorique, à ceux pour qui les choses sont blanches ou noires, orientales ou occidentales. Je feuilletai le reste du cahier... » (A. M., p. 153)

9 Tout le récit repose, en effet, sur ces rapports de domination entre l'Orient et l'Occident qui hantent les personnages. Les rencontres amoureuses entre Mustapha Saïd et les femmes européennes sont fatalement rattrapées par ce clivage qui assigne à chacun des 
partenaires un rôle défini à l'avance, à partir des représentations que chacun se fait de l'autre.

10 Pour s'adapter à un imaginaire occidental fait de stéréotypes et de fantasmes, Mustapha Saïd construit un espace oriental sur mesure, créé de toutes pièces pour plaire à des partenaires assoiffées d'exotisme en tout genre. Placée dans ce contexte, la chambre faussement orientale délocalisée à Londres, convoque ironiquement la nomenclature d'un tableau orientaliste où s'entassent tous les objets qui renvoient à l'Orient. Le personnage condamnant chacune de ses compagnes au suicide, cette chambre devient le théâtre de crimes perpétrés par un homme sauvage, qui, conformément aux fantasmes de ses concubines, doit assouvir ses désirs les plus primitifs en se montrant despote et sanguinaire ${ }^{6}$. Malgré l'importance symbolique de ce lieu, aussi bien sur le plan narratif que discursif, Fadi Noun décide tout simplement d'effacer tout le passage décrivant la chambre et ses différentes fonctions :

À Londres, je l'emmenai dans ma chambre, démenti aux illusions. Je la fis pénétrer dans un autre décor, menteur, mais celui-ci terrifiant et mortel. Elle s'agenouilla à mes pieds, me baisa et déclara: "Je fais soumission Moustafa! Mon maitre et seigneur. Fais de Suzanne ton esclave, ce que bon te semble! (F. N., p. 113)

11 Il faut attendre la seconde version d'Abdelwahab Meddeb pour que le lecteur francophone découvre la condamnation de ces représentations orientalistes que Tayeb Salih choisit de faire passer par la fiction, bien avant la parution de l'essai d'Edward Saïd :

À Londres, je l'emmenai dans ma chambre, repaire de tromperies scandaleuses que j'avais édifié sciemment, mensonge second. Santal, ambre, plumes d'autruches, statues d'ivoire, d'ébène, photos, images représentant des palmeraies du Nil, felouques sur l'eau, aux voiles comme ailes de colombe, soleils tombant dans la mer rouge à travers monts, caravanes de chameaux forçant la marche sur les dunes de sable aux confins du Yemen, baobabs de Kordofan, jeunes filles nues des tribus Zandi, Nuer, Choulouk, champs de bananiers et de caféiers sur la ligne de l'équateur, vieux temples pharaoniques de Nubie, couvertures de livres arabes embellies de caractères coufiques fleuris, tapis persans, rideaux roses, grands miroirs sur les murs, lumières multicolores dans les coins. Elle se prosterna, elle baisa mes pieds et déclara: «Toi Moustafa, tu es mon maître et seigneur, et moi, Suzanne, je suis ton esclave... » (A. M., p. 148-149)

Dans d'autres endroits, Fadi Noun va jusqu'à supprimer un certain nombre de références intertextuelles renvoyant à l'Orient, qu'il soit religieux ou encore littéraire. Dans l'exemple suivant, il fait le choix de ne pas traduire un des hadiths («dits» du prophète Mohamed) qu'Abdelwahab Meddeb rétablira dans la seconde version, que voici :

«Nous sommes un peuple coupé du monde. Contez-nous des histoires qui nous servent de parure... » Qui dit cela? "Comme un être résolu à vivre coupé de tout, mais il est incapable de franchir les distances quand même il n'aurait pas épargné les montures. » Le chauffeur ne dit mot. Il prolongeait la voiture qu'il maîtrisait. Il l'insultait parfois. Et la terre autour de nous était un cercle englouti dans le mirage. "Élevés par les uns, rabaissés par les autres, nous sommes un peuple rejeté de désert en désert. » (A. M., p. 112)

Manifestement agacé par la dimension intertextuelle qui fait toute l'originalité de l'écriture de Tayeb Salih, Fadi Noun décide, dans un autre passage, de ne pas traduire des paroles citées par le narrateur et attribuées à l'imam Al-Asmaï'. Encore une fois, c'est dans la seconde version que l'on peut de nouveau lire la citation : 
Celui qui enfante le bien donne au monde des oiseaux de joie. Et celui qui enfante le mal sème un arbre dont les jeunes épines sont le regret, et le fruit le remords. Que Dieu ait dans sa miséricorde un homme qui ne tenait pas compte des péchés et qui jouissait de l'Apparence. (A. M., p. 155)

14 Ces manipulations atteignent leur comble quand il s'agit de citer la poésie arabe classique. Réputée pour sa transgression des mœurs conservatrices, puisqu'elle se plaît à chanter ouvertement la beauté des éphèbes et les délices du vin, la poésie bachique a été remarquée très tôt par les orientalistes ${ }^{8}$. Dans le roman de Salih, elle joue un rôle important en lien avec l'histoire de l'orientalisme. En effet, pour charmer ses concubines, Mustapha Saïd se réfère volontairement à ce patrimoine littéraire en "tissant de fil à fil d'agréables chimères" (A. M., p. 148) afin de créer l'ambiance exotique d'un Orient fait de "mirages qui vibrent dans les brûlants déserts » (A. M., p. 48). Or, en confrontant la version de Fadi Noun avec la traduction d'Abdelwahab Meddeb, on se rend compte rapidement que certains vers ont été fusionnés sans aucun respect du texte source. Plus important encore, plusieurs passages clés manquent :

Sois heureux mon bien aimé de voir la terre fleurie de roses

et le vin vert s'offrir à toi comme une vierge insolente.

Ne boire ce vin résineux te laisse impardonnable,

Hâte-toi, car les jardins du Karkh sont riants de la nuit

Ici oubliés de la main gauche de la guerre.

Je récitai encore :

Abou' 1 Haïja a lancé ses cavaliers dans la bataille.

L'étendard déployé de la mort s'est déclaré au vieillard.

Et quand la violence fit rage, nos arcs à nous furent

Nos mains nues, les champs de lys nos flèches,

Notre guerre s'approfondit d'intimité confiante,

Mourir de plaisir devint sacré aux jeunes garçons charmés

Par la musique du luth faisant écho au tambour de bataille.

Échanson, on se dispute tes mains

Qui nous abreuvent de vin captif.

La guerre sans ennemi qui sait tuer à plaisir

À plaisir te fait renaitre comme et quand bon lui semble. (F. N., p. 112-113)

Ne te plait-il pas que la terre soit en fleurs

et le vin à la portée vieux et vierge

rien n'excuse ton abstinence c'est un vin mûr

fils de la nuit et de la verte vigne

hâte-toi car les jardins charmants de Karth

sont à l'abri d'une main rude à la guerre.

Ou encore :

J'ai bu une coupe, lanterne céleste

après un baiser ou une galante promesse

puis les jours se sont succédé

chutes de lumière à travers des déchirures du ciel.

Ou encore :

Quand le condottiere lança ses cavaliers dans le chaos de la mêlée

quand l'étendard de la mort se déploya devant le vieillard

quand la bataille fit rage

quand le feu dévasta les lieux

nos mains nues furent nos arcs, les lys nos flèches

notre guerre à nous est une intimité confiante

rassemblant des amis proches

quand les soldats battaient leurs tambours

par nos luths nous répondions

à des éphèbes qui voyaient la mort venir dans le plaisir 
la cause de notre guerre est un échanson qui nous sert

un vin captif nous incitant à boire

pour que les vivants rejoignent les morts

tu vois l'un terrassé l'autre ivre affalé

notre guerre est une guerre qui ne répand pas le malheur

elle ressuscite ceux qu'elle a jetés dans la mort. (A. M., p. 147-148)

Ces libertés prises dans le choix des mots et des passages à traduire ne fait que réduire l'importance du message véhiculé par ces poèmes par rapport à la problématique générale de l'œuvre: la poésie bachique et homoérotique d'Abū Nuwās révèle le raffinement et la liberté des mœurs dans les sociétés arabes à un moment donné de leur histoire. Ces extraits s'inscrivent en contre-point des fantasmes occidentaux réduisant l'étudiant soudanais à un être primitif et sauvage qui a besoin de s'élever socialement et culturellement. De surcroît, le mot " guerre » repris ici confronte in fine deux types de guerre : celle d'un Occident oppresseur menant une expansion coloniale au nom de la "civilisation pour tous " et celle des poètes arabes classiques luttant contre les ennemis du vin et des plaisirs libertins. Le décalage entre les deux guerres témoigne encore une fois de l'absurdité de la propagande coloniale et de ses ravages. Le verdict tombe quelques pages plus loin quand Fady Noun fait le choix de supprimer un magnifique compte-rendu de lecture que fait le personnage principal à partir d'un numéro du Times. Ce long passage donne des "nouvelles de l'Empire et de l'étranger » (A. M., p. 152) et révèle les faces cachées du colonialisme et de l'impérialisme anglais avec la complicité de quelques dirigeants du Moyen-Orient. Voici un extrait de ce qui n'a pas été retenu par Fady Noun :

Les nouvelles de l'Empire et de l'étranger. Nouvelle proposition de Moscou pour rembourser la dette russe à la France. Inondations en Suisse. Le Discovery, bateau du capitaine Scott, est de retour des mers méridionales. Herr Stresemann prononça un discours sur le désarmement à Genève, le samedi ; dans une interview le matin, il a aussi approuvé le discours de von Hindenbourg à Tannenberg qui refuse d'attribuer à l'Allemagne la responsabilité du déclenchement des hostilités. L'éditorial est consacré au pacte de Djeddah cosigné par Sir Gilbert Clayton, représentant de la Grande-Bretagne, et l'émir Fayçal Abdel Aziz Séoud, au nom du père roi du Hedjaz et de Nedjd. (A. M., p. 152-153)

Le traducteur se justifie au cœur même du texte traduit de la manière la plus éhontée : "Je parcourus le sommaire qui avait l'attrait de vieilleries exotiques; très vite pourtant il me lassa. Je n'y trouvai rien de significatif» (F. N., p. 115). Le lecteur se trouve induit en erreur, croyant qu'il s'agit de la voix du narrateur lui-même. Or, cette phrase est inexistante dans le texte source. C'est le traducteur en personne, si l'on peut dire, qui intervient directement et à l'insu du lecteur pour juger de l'insignifiance d'un passage pourtant clé pour comprendre les liens entre culture et impérialisme - pour emprunter les mots d'un titre d'Edward Saïd.

17 La suppression volontaire des références concerne également la bibliothèque occidentale. En effet, plusieurs auteurs et livres cités ou encore lus par le personnage principal du roman sont absents dans la première version en français. Abdelwahab Meddeb rétablit tous les noms manquants que nous signalons ici en gras :

Même si je réclame vengeance, je ne puis résister à la curiosité. Je verrai d'abord, et j'écouterai, puis je mettrai le feu; tout cela brûlera; comme s'il n'avait jamais existé. À la lumière de la lampe, je vis tant de livres alignés, bien rangés : livres d'économie, d'histoire, de littérature. Zoologie. Géologie. Mathématiques. Astronomie. L'Encyclopedia britannica, Gibbon, Macaulay, Toynbee, les œuvres de Bernard Shaw, Keynes. Tawney. Smith. Robinson, Un essai d'économie 
marxienne... Sociologie, anthropologie, psychologie. E.G. Moore. Thomas Mann, Thomas Hardy, Thomas Moore, Virginia Woolf, Wittgenstein, Einstein, Brierly. Namier. Des livres célèbres, d'autres inconnus. Des recueils de poètes dont j'ignorais l'existence. Le journal de Gordon, Les voyages de Gulliver. Kipling. Housman. L'Histoire de la Révolution française, Thomas Carlyle. Conférences sur la Révolution française, Lord Acton. Des livres reliés plein cuir, des livres recouverts de papier glacé, des livres usés, d'autres intacts comme sortis à l'instant de l'imprimerie, d'énormes volumes, de la taille des stèles funéraires, de petits livres dorés sur tranche au format d'un jeu de cartes. Des signatures, des dédicaces, des livres en caisse, par terre, sur les chaises. Quelle plaisanterie. Owen. Madox Ford, Stefan Zweig, E.G. Brown. Laski. Hazlitt. Alice au pays des merveilles, le Coran et les Évangiles en anglais, Gilbert Murray. Platon. L'Économie coloniale, Moustafa Saïd, La croix et la Poudre à canon, Moustafa Saïd, Le pillage de l'Afrique, Moustafa Saïd, Prospéro et Caliban, Totem et Tabou. Doughty. Pas un livre arabe. Un tombeau. Un mausolée. Une idée folle. Une grande farce. Une prison. Un trésor. Sésame ouvre-toi et que l'on distribue ces pierres précieuses à tous les présents. » (A. M., p. 139-140)

Cette bibliographie d'une grande richesse témoigne des lectures de Mustapha Saïd, en lien avec le colonialisme, l'orientalisme et l'impérialisme anglais. Or, il suffit de parcourir les index de l'Orientalisme (1978) et de Culture et impérialisme (2000) d'Edward Saïd pour découvrir qu'une grande partie des références qui manquent dans la traduction française de 1972 figure aussi bien dans le texte arabe de 1969 que dans les deux livres de Saïd datant respectivement de 1978 et de $2000^{\circ}$. Saïd a-t-il lu les versions arabe et anglaise de Saison de la migration vers le Nord, avant la rédaction de L'Orientalisme? Il est difficile de le confirmer, toutefois, Tayeb Salih est clairement cité dans Culture et Impérialisme :

Dans d'autres cas, des romanciers comme le Kenyan Ngugi et le Soudanais Tayeb Salih s'approprient dans leur œuvre de fiction de grands topoi de la culture coloniale comme la quête et le voyage dans l'inconnu: ils les revendiquent au service de leurs propres projets postcoloniaux. Le héros de Salih dans Saison de la migration vers le Nord fait (et est) le contraire de ce que fait (et est) Kurtz. L'homme noir voyage vers le nord et s'enfonce en territoire blanc ${ }^{10}$.

19 En somme, à force de gommer les formules prétendument exotiques, Fady Noun a fini par nettoyer le texte arabe de toute forme de critique pouvant atteindre l'héritage orientaliste, allant jusqu'à occulter des références intertextuelles et bibliographiques nécessaires à tout travail d'historicisation intellectuelle. Ainsi, le roman de l'écrivain soudanais ne figure dans aucune bibliographie, du moins française, le désignant comme l'un des précurseurs de L'Orientalisme d'Edward Saïd ${ }^{11}$.

Se situant en contre-point de la posture adoptée par Fadi Noun, Abdelwahab Meddeb, tel un véritable passeur de langues et de cultures entre l'Orient et l'Occident, nous propose une traduction qui non seulement reconstruit un texte tronqué mais surtout inscrit la littérature arabe contemporaine dans les débats postcoloniaux qui vont se diffuser après la publication de L'Orientalisme. Telle est l'orientation que Abdelwahab Meddeb donnera, entre autres, à son engagement intellectuel: relire les rapports complexes entre Orient et Occident dans le sillage de Tayeb Salih et Edward Saïd, sans pour autant tomber dans une lecture manichéenne enfermant le Nord dans une forme d'hégémonie diabolique et le Sud dans une subalternité sans issue. 


\section{Vers une histoire des migrations intellectuelles}

Abdelwahab Meddeb a rendu l'œuvre de Tayeb Salih à la critique de l'orientalisme qui la fondait et on peut supposer que sa propre œuvre s'est nourrie de cette traduction. Nous voudrions, pour conclure, esquisser des pistes, identifier des lieux de convergence entre les deux œuvres, en particulier autour du motif de la hijra et du procédé de tissage de différents textes.

Il est frappant de voir combien l'expression hijra est chère à Abdelwahab Meddeb dont l'œuvre est rythmée par des réflexions autour de l'exil, du déplacement, des tours et des détours. Cette expression qu'il a eu l'occasion de traduire par « migration » dans le titre du roman de Tayeb Salih revient régulièrement sous sa plume, en lien avec d'autres réalités, par exemple dans son récit Talismano réédité en 1983: «Maintes sorcières et autres énergies tenaces se sont séparées avec nous de la ville. Hijra, migration volontaire, à suivre le chemin de Hâjir, Agar, hors défaite, vers le retrait : orphelins ${ }^{12}$ ». Au-delà des relations conflictuelles entre Orient et Occident que le récit de Tayeb Salih met largement en scène, Meddeb réalise un dépassement en proposant d'autres formes de migrations : la Hijra concernera désormais chez lui la circulation des langues et des savoirs entre Orient et Occident. Pratiquement toute son œuvre est tissée de ces « errances » intellectuelles qui mettent à l'épreuve les représentations monolithiques des cultures. Ce dépassement, Meddeb le formulera à partir de sa traduction de L'Exil occidental, le récit du philosophe mystique iranien Suhrawardi qu'il publie en $199 \overline{\overline{6}}$ :

Lorsque je consulte l'entrée h.j.r., verbes et substantifs se succèdent pour dire le départ, la rupture, l'abandon, le délaissement, l'éloignement. L'on voyage et se sépare des siens; ;'on émigre en quittant sa tribu, délaissant le désert et le mode de vie nomade pour s'établir en ville. C'est le départ d'un pays vers un autre qui donnera hijra' ${ }^{13}$.

D'ailleurs, Abdelwahab Meddeb reconnaît l'influence de cette traduction sur un parcours marqué par l'exil et la migration : «Quand j'ai lu, il y a plus de vingt ans, pour la première fois, Le Récit de l'exil occidental, j'ai eu le sentiment que ce texte m'était personnellement adressé. [...] Dès le titre, l'expression d'exil occidental cristallisait mon expérience ${ }^{14} »$.

La même thématique, en lien avec les débats postcoloniaux qui s'installent peu à peu en France, fera l'objet d'un colloque organisé, en 1996, par la revue Dédale qu'il dirige et le Musée national des arts d'Afrique et d'Océanie. Les actes du colloque, publiés en 1997, portent un titre symbolique : Postcolonialisme, Décentrement, Déplacement, Dissémination ${ }^{15}$. Le texte d'ouverture de Sami Naïr, intitulé «Les deux regards » résonne avec le roman de Tayeb Salih qui aborde les mêmes problématiques :

Entre le Nord et le Sud de la Méditerranée, il s'agit d'abord d'un problème de représentations. Il y a bien sûr l'inégalité des richesses, la diversité des modes d'organisation sociale, la distribution différenciée des statuts sociaux et des sexes. Mais il y a plus: il y a surtout la manière dont les deux rives se perçoivent. Perception trouble, qui fonctionne autant à la raison qu'au sentiment; une sorte de réflexe à la fois spontané et contrôlé, impulsif et réflexif, qui provoque ou l'angoisse ou la haine, la compassion ou l'indifférence ${ }^{16}$.

De toutes ces formes de migrations se construit peu à peu un réseau de significations complexes visant à redéfinir l'Orient et l'Occident à partir d'une relation rhizomatique déniant toute hiérarchie entre les deux cultures. Cette circulation des savoirs se déploie chez Abdelwahab Meddeb grâce à la traduction de citations qui instaure, au sein même 
de la narration, un dialogue fécond entre plusieurs patrimoines littéraires et artistiques.

Dans plusieurs de ses récits, l'acte de traduire est indissociable de l'acte de l'écriture. Fidèle à l'hétérogénéité, à la fois générique et scripturale, qui faisait l'originalité des romans de Tayeb Salih, Abdelwahab Meddeb poursuit ce dialogue en traduisant les mêmes poètes que l'écrivain soudanais. En témoigne, par exemple, la présence du poème bachique d'Abū Nuwās traduit dans Phantasia publié en 1986 :

Apprivoise l'indomptable liqueur fauve.

Coupe-la d'eau si tu la vois à ton geste soumise,

arrête là ta main ; qu'elle ne rende pas l'âme.

Conserve son esprit pour l'harmonie des vivants.

Jaunâtre dès qu'elle paraît elle abolit le malheur

et prête au cœur une parure de joie.

Elle tremble d'un diadème qu'ornent

deux lignes écrites d'une main gauche.

Elle répand sa brise sur les commensaux

et éclaire dans la nuit noire.

Quand je l'ai surprise impatiente dans la coupe

et oppressée telle une vierge pubère, j'ai dit :

il faut mordre dans ses lèvres et la contenter ;

que mes entrailles se mêlent aux siennes.

Svelte et élancée aux sens je l'ai éveillée.

Enfin apaisée les yeux clos elle s'est assoupie, et sa langue ivre à moi s'est plainte

en un balbutiement de bègue.

Je l'ai consolée tandis qu'au cœur brûlait

une passion, flammes embrasant l'herbe sèche.

Je n'ai pas traduit, dans ce poème d'Abū Nuwās, vin par liqueur, dans la seule intention de réhabiliter un langage poétique désuet, dont les vocables renaissent à la beauté, quant, profitant du déclin de la polémique qui les as bannis, je les arrache du site qui les as flétris. Cette interprétation me procure un mot féminin, équivalent à l'original, et capable de parcourir le trajet de l'étrangeté de la métaphore érotique, même si cette option accentue l'étrangeté de la description, par égard au champagne. Ce poème aurait mieux convenu à un vin liquoreux et amélioré par les Arabes. Mais cette évocation ne sera plus incongrue si l'on admet qu'elle traque avec éloquence l'opération que trame le vin dans le corps ${ }^{17}$.

S'inscrivant parfaitement dans la continuité des fragments poétiques retraduits par Abdelwahab Meddeb dans Saison de la migration vers le Nord, le recours à Abū Nuwās comme figure tutélaire de la poésie bachique va au-delà de la simple citation d'un patrimoine littéraire arabe méconnu. Plus important encore, pour Tayeb Salih, comme pour Abdelwahab Meddeb, la citation relève d'un engagement politique plus large dont l'objectif est de combattre une idéologie islamiste conservatrice qui a déclaré la guerre à toutes les formes du plaisir terrestre comme le vin et la poésie bachique. Rappelons, à cet égard, que Saison de la migration vers le Nord a été la cible du gouvernement soudanais qui déclara, en 1996, les sujets traités par le roman comme contraires aux bonnes mœurs du peuple soudanais ${ }^{18}$. Malgré son succès partout dans le monde, le livre a été retiré du programme littéraire au Soudan.

Pour finir, il est important de rappeler qu'Abdelwahab Meddeb n'a jamais complètement coupé avec l'œuvre de Tayeb Salih dont la traduction a été confiée 
depuis à Anne Wade-Minkowski. En effet, Abdelwahab Meddeb a continué à fréquenter l'œuvre en tant que relecteur. En témoigne par exemple le remerciement que lui réserve Anne Wade-Minkowski, dans sa traduction du roman Bandarchâh, publié chez Sindbad en 1985 :

Je voudrais enfin adresser mes remerciements à l'auteur qui m'a aidée dans la compréhension d'un dialecte sensiblement distinct de ceux que l'on entend en d'autres pays arabes, et à Abdelwahab Meddeb dont la lecture attentive et exigeante m'a été un précieux soutien ${ }^{19}$.

Il ne s'agit en aucun cas de forcer une quelconque filiation entre Salih et Abdelwahab Meddeb; il s'agit plutôt d'identifier des résonances, des réminiscences, des traces épiphaniques si l'on se réfère au vocabulaire mystique cher à Abdelwahab Meddeb. Ce large réseau de branchements ${ }^{20}$ relie non seulement des littératures d'Orient et d'Occident mais encore des genres culturellement hétérogènes, sans cesse retravaillés par la traduction. C'est enfin, dans cette même perspective, que l'œuvre de Tayeb Salih, comme celle de Abdelwahab Meddeb, peut être lue comme un véritable espace d'écarts féconds et de dialogue, dans le sens que François Jullien donne à ces notions, loin de l'usage un peu trop galvaudé de nos jours :

En posant à la fois le dia de l'écart: un dia-logue est d'autant plus riche, nous montraient déjà les Grecs, qu'il met de l'écart en jeu et permet de la confrontation ; et le logos de l'intelligible : il n'y a d'ineffable culturel de quelque culture que ce soit, même de la "sinité ", et toutes les cultures sont appelées non pas au compromis je refuse cette façon timorée d'entendre la tolérance - mais à se comprendre. C'està-dire, au travers d'écarts qui ne cessent de se configurer, à relancer l'intelligence du commun ${ }^{21}$.

\section{NOTES}

1. T. Salih, موسم الهجرة إلى الشمال (Saison de la migration vers le Nord), Beyrouth, Dar Al-'Awdah, 1966.

2. T. Salih, D. Johnson-Davies (trad.), Season of Migration to the North, Londres, Heinemann edition, 1969.

3. Parue en 1972 chez Sindbad, la première version, traduite par F. Noun sous le titre Le Migrateur, est introduite par une préface de J. Berque qui ne sera pas reprise dans la seconde version du roman proposée, en 1983, par A. Meddeb sous le titre Saison de la migration vers le Nord, chez Sindbad. Les références aux deux versions seront mentionnées dans le corps du texte, respectivement par les abréviations F. N. et A. M.

4. Th. Brisson, «L'Occident / Orient comme enjeux épistémiques: crise du tiers-mondisme, connexions transnationales et déterminations politiques des critiques arabes (Abdel-Malek, Alatas, Patterjee) ", dans Les Occidents des mondes arabes et musulmans, M. Del Fiol et Cl. Cécile Mitatre (dir.), Paris, Geuthner, 2018, p. 115-140.

5. A. Abdel-Malek, « L'orientalisme en crise », Diogène, $\mathrm{n}^{\circ}$ 44, $4^{\mathrm{e}}$ trimestre, 1963, p. 109-142.

6. Le procès de Mustapha Saïd, à la fin du roman, est particulièrement éloquent : le juge explique la monstruosité de l'étudiant soudanais par les crimes du colonialisme. Autrement dit, selon le juge c'est l'homme blanc qui a fabriqué, à partir de ses fantasmes et ses représentations, une créature primitive monstrueuse qu'il a appelée "Africain» afin de justifier son entreprise 
civilisatrice. Il est frappant de voir à quel point ces arguments dialoguent en profondeur avec les propos de Jean-Paul Sartre dans sa préface des Damnés de la terre de Frantz Fanon: "L'élite européenne entreprit de fabriquer un indigénat d'élite ; on sélectionnait des adolescents, on leur marquait sur le front, au fer rouge, les principes de la culture occidentale, on leur fourrait dans la bouche des bâillons sonores, grands mots pâteux qui collaient aux dents. [...] C'était l'âge d'or. Il prit fin: les bouches s'ouvrirent seules; les voix jaunes et noires parlaient encore de notre humanisme mais c'était pour nous reprocher notre inhumanité. [...] Une autre génération vint, qui déplaça la question. Ses écrivains, ses poètes, avec une incroyable patience, essayèrent de nous expliquer que nos valeurs collaient mal avec la vérité de leur vie, qu'ils ne pouvaient tout à fait ni les rejeter ni les assimiler. En gros, cela voulait dire: vous faites de nous des monstres, votre humanisme nous prétend universels et vos pratiques racistes nous particularisent.» (Préface de l'édition de 1961, dans Fr. Fanon, CEuvres, Paris, La Découverte, 2011, p. 431-432).

7. Philologue et grammairien de Bassora, l'imam Al-Asmaï (740-828) est connu surtout pour ses travaux scientifiques en zoologie et en botanique. Considéré comme un grand classique de la vie intellectuelle arabe, ses propos sont souvent repris par des philosophes, comme c'est le cas ici. En effet, les paroles tronquées dans la version de Fadi Noun sont citées par le philosophe arabophone Al-Jahiz dans son livre Bayān wa-al-tabyīn.

8. A. Nuwās est mentionné pour la première fois comme poète arabe classique dans le Catalogue de la bibliothèque orientale de feu M. Jules Thonnelier, orientaliste, membre de la société asiatique et de la société de l'histoire de France, édité à Paris par Ernest Leroux en 1880. Il est également cité dans Les Manuscrits arabes de l'Escurial I: Grammaire, rhétorique, poésie, philologie et belles-lettres, lexicographie, philosophie, de H. Derenbourg, premier volume édité entre 1884 et 1903 par E. Leroux, dans la collection «Publications de l'École des langues orientales vivantes ».

9. Nous avons relevé dans Orientalism les noms suivants: Owen, Roger, 327, 356, 374, 376, Smith, William Robertson, 234-6, 238, 270, 277, 370-71, Hobson, J. A., 92, Macaulay, Thomas Babington, 14, 152, 196, 366, Moore, Thomas, 118. Dans Culture et impérialisme, Saïd cite Hobson, J.A, 39, 48, 124, 140, 170, 244, 270, 316, 340. Impérialism, 340, Macaulay, Thomas Babington, 133,160, 165, 172, 202, 283. Minute on indian Education, 160, Owen, Roger, 194, 195, Robinson, Ronald, 40, 127, 366, 367, Smith, Bernard, European Vision and the South Pacific, 16, Thomas Hardy, 202, 203, 231, 231, 232, 270. Jude l'Obscur, 232, 233, Gordon, Charles (général), 174.

10. E. Saïd, Culture et impérialisme, Paris, Fayard/Le Monde diplomatique, 2000, p. 71.

11. Ce n'est pas la première fois qu'un traducteur arabisant fait le choix de ne pas traduire de l'arabe une critique ouverte des Orientalistes. Par exemple, dans sa traduction du récit de voyage arabe La Jambe sur la Jambe de A. Faris ach-Chidyâq, R. Kawam fait le choix de supprimer un supplément adressé « aux Orientalistes détenteurs de chaires en arabe dans les grandes écoles de Paris ». Cette postface que j'ai traduite en collaboration avec A.AbelKader, est désormais disponible dans Les Orientaux face aux orientalismes, R. Boulaâbi (dir.), Paris, Geuthner, 2013.

12. A. Meddeb, Talismano, Paris, Sindbad, 1983, p. 241.

13. A. Meddeb, L'Exil occidental, Paris, Albin Michel, 1993, p. 72.

14. Ibid., p. 71.

15. Postcolonialisme, Décentrement, Déplacement, Dissémination, Dédale, $\mathrm{n}^{\circ} 5 / 6$, Paris, Maisonneuve et Larose, 1997.

16. Ibid., p. 17.

17. A. Meddeb, Phantasia, Paris, Sindbad, 1986, p. 138-139.

18. Dans son article publié dans le quotidien soudanais Alhayat, daté du 26 août 1996, le journaliste Talha Jibril s'indigne contre l'interdiction du roman de Tayeb Salih du programme officiel et explique les causes profondes d'une telle censure. Voir en ligne: <http:// www.alhayat.com/article/1886392>.

19. A. Wade-Minkowski (trad.), Bandarchâh, Paris, Sindbad, 1985. 
20. J.-L. Amselle, Branchements. Anthropologie de l'universalité des cultures [2001], Paris, Flammarion, Champs, 2005.

21. Fr. Jullien, L'Écart et l'entre: Leçon inaugurale de la Chaire sur l'altérité, Paris, Galilée, 2012, p. 46-47.

\section{RÉSUMÉS}

À partir d'une étude comparative de deux traductions en français de موسم الهجرة إلى الشمال (Saison de la migration vers le Nord), un grand classique de la littérature arabe des années soixante de l'écrivain soudanais Tayeb Salih, cet article se propose de montrer comment certains choix pris par les traducteurs révèlent des postures qui vont au-delà du simple fait de traduire. En effet, au cœur de l'espace du texte traduit, se joue une bataille en lien avec la discipline orientaliste. Si la première version de Fady Noun offre au lecteur français une vision complètement édulcorée de l'imaginaire oriental sur l'occident, gommant presque systématiquement les critiques de l'orientalisme qui rythment le texte arabe, la seconde version d'Abdelwahab Meddeb rétablit les passages coupés et permet de se rendre compte du rôle important joué par la fiction arabe dans la déconstruction du savoir orientaliste, quelques années avant la publication de Orientalism d'Edward Saïd, en 1978. En replaçant les deux versions dans le contexte intellectuel français et européen avant et après le tournant saïdien, cette étude ambitionne, d'une part, de contribuer à la relecture de l'héritage orientaliste à partir du domaine arabe qui a certainement nourri la critique de Saïd. D'autre part, elle propose des pistes de réflexion sur les répercussions de ces débats que révèle le texte traduit sur l'œuvre littéraire d'un écrivain et essayiste comme Meddeb.

Based on a comparative study of two French translations of Season of migration to the North, a classic of Sudanese writer Tayeb Salih's Arab literature of the 1960s, this article aims to show how certain decisions taken by translators reveal postures that go beyond the simple fact of translating. Indeed, at the heart of the space of the translated text, there is a battle going on in relation to the orientalist discipline. If Fady Noun's first version offers the French reader a completely watered-down vision of the oriental imagination on the West, almost systematically erasing the criticisms of orientalism that punctuate the Arabic text, Abdelwahab Meddeb's second version restores the cut passages and makes it possible to realize the important role played by Arab fiction in the deconstruction of orientalist knowledge, a few years before Edward Said's publication of Orientalism, in 1978. By placing the two versions in the French and European intellectual context before and after the Said turn, this study aims, on the one hand, to contribute to the re-reading of the orientalist heritage from the Arab field, which has certainly nourished Said's criticism. On the other hand, it offers avenues for reflection on the repercussions of these debates that the translated text reveals on the literary work of a writer and essayist like Meddeb. 


\section{AUTEUR}

\section{RIDHA BOULAÂB}

Université Grenoble Alpes ; UMR 5316 Litt\&Arts

Né en 1978 à Thala (Tunisie), Ridha Boulaâbi est maître de conférences en littératures

francophones à l'Université Grenoble Alpes et actuellement chargé de mission francophoniesrelations internationales. Ses travaux de recherche portent sur l'altérité linguistique et culturelle dans les œuvres de Claude Ollier, Gérard Macé, Roland Barthes, Louis Aragon, Abdelwahab Meddeb, Kateb Yacine, Abdellah Taïa, Alaa Al-Aswany... Il s'intéresse également aux récits de voyage en Occident des écrivains arabes du XIX ${ }^{\mathrm{e}}$ siècle comme Rifaâ Tahtawî, Faris Ach-Chidyâq, Al-Afghâni et prépare une étude sur les rapports entre la francophonie et l'orientalisme. Il est l'auteur de L'Orient des langues au XXe siècle: Aragon, Ollier, Barthes, Macé, Paris, Éditions Geuthner, 2011 ; Nedjma de Kateb Yacine, Champion, coll. « Entre les lignes/littératures Sud », Paris, 2015. Il a également dirigé un ouvrage sur Les Orientaux face aux « orientalismes », Paris, Éditions Geuthner, 2013. 